\title{
The Effects of Different Music Genres on Heart Rate Variability in Extremely and Very Low Birth Weight Preterm Neonates: an Italian Randomized Pilot Study
}

\section{Barbara Sgobbi}

ASST dei Sette Laghi: Aziende Socio Sanitarie Territoriale dei Sette Laghi

\section{Maria Elena Bolis}

ASST dei Sette Laghi: Aziende Socio Sanitarie Territoriale dei Sette Laghi

Laura Morlacchi ( $\sim$ laura.morlacchi@asst-settelaghi.it)

ASST dei Sette Laghi: Aziende Socio Sanitarie Territoriale dei Sette Laghi

Jennifer Meessen

ASST dei Sette Laghi: Aziende Socio Sanitarie Territoriale dei Sette Laghi

Alfredo Raglio

Maugeri Clinical Research Institutes IRCCS Pavia: Istituti Clinici Scientifici Maugeri SpA IRCCS Pavia

Massimo Agosti

ASST dei Sette Laghi: Aziende Socio Sanitarie Territoriale dei Sette Laghi

\section{Research}

Keywords: music listening, music genres, NICU, preterm neonates, heart rate variability

Posted Date: March 8th, 2021

DOl: https://doi.org/10.21203/rs.3.rs-286025/v1

License: (c) (i) This work is licensed under a Creative Commons Attribution 4.0 International License.

Read Full License 


\section{Abstract}

Background: Music and music therapy, live or recorded, has been gradually introduced in the Neonatal Intensive Care Unit (NICU) of our Hospital in addition to standard medical and nursing care, to improve premature neonates' neurophysiological development. The present study focused on music genres, starting from the parents' sound experience and musical tastes that neonates experienced during the pregnancy period. The study explored the effects of different music genres also on Extremely Low Birth Weight (ELBW) neonates since most studies refer to Very Low Birth Weight (VLBW) neonates with birth weight $>1000 \mathrm{gr}$. The study aims to investigate the influence of different music genres on Heart Rate Variability (HRV) of preterm neonates.

Methods: a prospective randomized study was conducted. Preterm neonates (gestational age $<32$ weeks, birth weight $<1500 \mathrm{~g}$ ), were recruited. On the basis of birth order, they underwent a daily randomized music listening program (25 minutes, every day, for 3 days) based on different music genres listening (soft poprap, pop-rock and classical music), followed by 1 day of no music monitoring (25 minutes). An electrocardiogram was performed to evaluate HRV parameters in each neonate with and without music exposure.

Results: twenty-seven (13 ELBW, birthweight <1000 g and 14 VLBW, birthweight 1000-1500 g) infants were included and HRV parameters were analysed. In the VLBW group no significant results were found between the music genres. In the ELBW group, during Soft Pop-Rap music listening, starting after 5 minutes High Frequency (HF) increase significantly (HF P $=0.0096)$ and Low Frequency $(L F)$ and LF/HF indices decrease significantly ( $L F P=0.0096 ; \mathrm{LF} / \mathrm{HF} P=0.0068$ ). The same trend was from 15 minutes of music listening high frequency (HF) increased and low frequency (LF) decreased (LF P=0.007; HF P= 0.007), Consequently LF/HF also decreased significantly (LF/HF $P=0.0093)$.

Conclusion: A significant decrease in the activity of the sympathetic and a simultaneous increase of the parasympathetic nervous system were found in the ELBW group during Soft Pop-Rap music listening. Further studies with a larger sample size are needed to confirm these findings and to elucidate the underlying mechanisms.

Trial registration: ClinicalTrials.gov Identifier: NCT04049526 The Effects of different music genres on heart rate variability in extremely and very low birth weight new-borns.

\section{Introduction}

Music and music therapy, live or recorded, has been gradually introduced in the Neonatal Intensive Care Unit (NICU) of our Hospital in addition to standard medical and nursing care, to improve premature neonates' neurophysiological development [1-9]. In most studies two types of music genre were commonly used: classical music $[2,5]$ and lullabies $[1,8]$. Other recent studies focused on the effects of the maternal voice or the entrained singing $[9,10]$ and live music $[3,8]$, highlighting their benefits on vital signs, sleep, body movements but also on not-nutritive sucking, on the caloric intake and on the parental 
stress. Recent literature suggests that parental involvement in live music session during Music Therapy sessions promotes direct interaction between preterm neonates and their parents. This approach can facilitate infant's development and parent's role $[9,10]$. The present study focused on music genres and explored the effects of different music genres also on Extremely Low Birth Weight (ELBW) neonates (birthweight $<1000 \mathrm{~g}$ ), since most studies refer to Very Low Birth Weight (VLBW) neonates with birthweight $>1000 \mathrm{gr}$. Indeed, only a limited number of studies have explored the benefit of music therapy for ELBW: Standley's meta-analysis [11] included only three studies evaluating infants $<28$ th Gestational Age without showing any significant effect of music therapy. It is also known that in the NICU environment the use of recorded music is more easily implemented inside incubators for certified music therapists than live music. The study aimed to investigate the influence of different music genres on Heart Rate Variability (HRV) of premature neonates. In particular, objective of the study was to observe neonates' responses in Heart Rate Variability (HRV) parameters to different music stimulations with the aim to identify the most effective therapeutic music approach in NICU.

\section{Materials And Methods}

This prospective randomized study was conducted in the Neonatal Intensive Care Unit of Filippo Del Ponte Hospital, Varese. The inclusion criteria were gestational age between the 23rd and the 32nd week, birth weight $<1500 \mathrm{~g}$ and appropriate for gestational age, sufficient stability of the vital parameters and absence of active infections and episodes of apnoea during the 72 hours before the treatment. Neonates with genetic anomalies, chromosomal aberrations, congenital heart malformations (except patent ductus arteriosus), intraventricular haemorrhage (> grade II on ultrasound) or periventricular Leukomalacia were excluded from the study. We subsequently excluded also the neonates with failed responses to bilateral hearing screening performed by Automated Auditory Brainstem Response testing (AABR). The study was performed within 15 days of birth after medical screening. The study was approved by the institutional research ethical committee of our Institution and neonates' parents gave written informed consent in accordance with the Declaration of Helsinki.

\section{Procedures}

Three playlists related to three different music genres (soft pop-rap, pop-rock and classical music) were used in the study, allowing to explore neonates' responses to different kinds of music in a standardized way. The duration of each playlist was 25 minutes, included 5 minutes of silence before the music, 15 minutes of music listening and 5 minutes of silence after the music. We used 15 minutes since in the literature of recorded music the administration was between 10 and 30 minutes of music listening [8]. Music listening was administered at the same time of the day (between 12p.m.-13p.m.), two hours after the day care (bath, cleaning, etc.) and before getting in contact with the parents. The study lasted four consecutive days. The first day baseline measurements were performed without music and in the following days the selected playlists were played in random order, one for each day (Fig. 1). 
A blinded researcher used a computerized random-number generator to assign each participant to one of the different music listening sequences and to randomize the order of songs in each sequence. The music was administered by a certified music therapist through speakers placed in the incubator. Acoustic methodology and monitoring followed Lubetzky's criteria [5]. Music was played using an Ipod and the speaker device was calibrated according to the American Academy of Pediatrics recommendations [12] (volume $<65 \mathrm{~dB}$ and background noise near the neonates' ear $<45 \mathrm{~dB}$ ). According to this acoustic measurement, speakers were placed inside the incubator at $30 \mathrm{~cm}$ from the neonates' ears, by adjusting the volume according to prematures' behaviour, heart rate and oxygen saturation. To monitor the noise and volume we used a professional sound level meter and lpod-volume (outside the incubator by hand). Internal noise in the incubators with premature baby inside and "C-Pap Ventilation" turned on were from $70 \mathrm{dBA}$ Medium and $80 \mathrm{dBA}$ Peak (international recommended threshold: average less than $50 \mathrm{dBA}$ peaks below $70 \mathrm{dBA}$ ). In order to eliminate background noise, the following measures were adopted: deactivation of monitors and alarms (only visual signal), all doors closed to minimize the noise from outside, staff invited not to talk over while the study was carried out.

\section{Assessment}

An electrocardiogram (ECG) was performed during each session of the study to evaluate the HRV in each neonate. HRV has been used to evaluate autonomic nervous system fluctuations in heart rate related to maturation in healthy neonates and in neonates with pathological conditions [13]. The HRV is more sensitive than other markers such as heart rate, respiratory rate, oxygen saturation and observational parameters to detect a change. This parameter was used to show how music could improve well-being and the development of the autonomic nervous system on autonomous stability through HRV measurement in premature baby. The ECG analogue signal from a heart rate monitor (mini cardio MC030, Hosand) was fed into a computer containing the HRV software (Train Me Coach, Hosand). The analogue ECG signal was converted to digital values reflecting cyclic changes in the RR interval. The sampling rate of the ECG recording device measures the ECG signal in $\mathrm{mV}$ and detects the peak $\mathrm{R}$ and stores the RR interval with $1000 \mathrm{~Hz}$ precision. The data were transformed into a waveform across a spectrum of various frequencies. Frequencies were measured in hertz $(1 \mathrm{~Hz}=1 \mathrm{cycle} / \mathrm{sec})$. Applied to HRV, sympathetic influences appear at frequencies $<0.15 \mathrm{~Hz}$, the low frequency (LF) power spectrum. The high frequency (HF) power spectrum is in the frequency range of $>0.15-0.40 \mathrm{~Hz}$, which is predominately influenced by parasympathetic inputs. The HRV indices for LF, HF, LF/HF ratio were analysed in different study's sessions. Stressor conditions were associated with an increase in LF, a decrease in HF power, and/or an increase in the LF/HF ratio. LF/HF ratio represents a general increase of the wellbeing when its value diminishes [14].

\section{Statistical analysis}

Baseline characteristics are presented by gestational age mean \pm SD or median (range) for continuous variables and numbers and percentages for nominal parameters. The normality of the data distribution was assessed by the Lilliefors (Kolmogorov-Smirnov) test for normality. Differences in response to each 
type of music as compared to baseline were tested by means of Wilcoxon test for paired data, for the whole cohort as well as for ELBW and VLBW separate. In addition, for each music genre the change in $\mathrm{LF} / \mathrm{HF}$ was calculated by subtracting the baseline measurement from the measurement during the 3rd song within each measurement. This difference was then compared with the change at the no-music day by means of Wilcoxon test for paired data. Differences were considered significant when $p<0.05$. The statistical analysis was performed by using MedCalcR for Windows, version 15.6.1 (MedCalc Software bvba).

\section{Results}

Thirty premature neonates were recruited in this study. We conventionally indicated as ELBW the enrolled infants with birthweight $<1000 \mathrm{~g}$ and as VLBW the enrolled infants with birthweight $1000-1500 \mathrm{~g}$. Three neonates enrolled in the study were excluded for incomplete data and unusable recording, thus data of 27 preterm neonates (13 ELBW and 14 VLBW), were analysed. Baseline demographic and clinical characteristics are summarized in Table 1.

No adverse events were noticed in this study. Music genres' characteristics and analysis are reported in Table 2.

Upon comparing music phases with baseline (no music), in the VLBW group no statistically significant results were found when different music genres were administered to infants (see Table 3). However, in the ELBW group, significant differences were found for Pop-Rap music: after 10-15 minutes the HF values increased significantly as compared to no music (9.2 to $16.7 \mathrm{HF}, \mathrm{P}=0.096)$ whereas the LF decreased significantly (90.8 to 83.3 LF, $\mathrm{P}=0.010)$. Consequently, the LF/HF decreased significantly from 14.4 to 7.3 UNIT $(P=0.007)$. None of the other music genres showed similar results (Table 4$)$.

\section{Discussion And Conclusion}

Unlike previous studies, this explorative research focused on the effects of pre-recorded music both in VLBW and in ELBW infants.

Soft Pop-rap music's administration showed, in the ELBW group, a significant decrease in the activity of the sympathetic nervous system and a simultaneous increase of the parasympathetic, generating a general state of stabilization of those parameters as reflected by LF/HF trend.

In a stressful environment such as NICU setting, music therapy could represent a valuable tool to improve premature neonates' wellbeing and to positively influence their neurophysiological development.

Particularly, the knowledge of which type of music genres can be more effective in reducing stress could suggest how to better combine music therapy with ordinary medical management of preterm neonates: for example, we would be able to know how to reduce their stress during potentially painful procedures and how to associate specific music genres' listening with the usual analgesic techniques. 
Focusing on the reasons of this positive effect of soft pop-rap compared to pop-rock music, it could be interesting to examine their musical structure. In this study, soft pop-rap music combines simple harmony, lower pitch and slower tempo, characterized by a constant, repetitive and predictable rhythmic, which could explain the relaxing and soothing effect on the premature neonates. Singer's voice used for soft pop-rap music genre has a much smaller extent with regard to the amplitude of the notes, it remains ever closer to the spoken voice "rap" and not "rock" melodic voice. This composition is very similar to the structure of the lullabies, unlike classical music which can be more complex for timber-harmonicrhythmic. The extremely preterm neonate is unable to discriminate complicated frames of tunes due to the immaturity of the nervous system, which does not allow him/her to process and integrate too complex sensory stimulation in the early stages of life [15]. In addition, the soft pop-rap music that we have administered is characterized by a male singing voice based on frequencies $(\mathrm{Hz})$ that could reflect the low tones of the uterus endogenous sounds and could therefore remind the child of the pre-natal sound environment.

Possible limitations of our study are the small sample size and the study design: a randomized controlled trial based on a music treatment could represent a more adequate methodological approach to investigate music effects in NICU and limit possible bias. In addition, in this research the different music genres were proposed to each neonate only once, but different reactions could have been observed after a second or third music administration [16].

Further randomized controlled trials (also comparing male/female voices and different kinds of prerecorded and live music) with larger sample size are needed to confirm these findings and to elucidate the underlying mechanisms of the music therapy effects on preterm infants.

Moreover, it will be useful to include other assessments (vital parameters, biological markers, etc.) and to extend the evaluation to neonates' parents in order to better understand the potential benefits of music therapy in NICU.

\section{Declarations}

\section{Ethics approval and consent to partecipate}

N. 51/2019 Comitato Etico dell'Insubria Varese Italy. Research involving human participants, human material, or human data, must have been performed in accordance with the Declaration of Helsinki and must have been approved by an appropriate ethics committee.

\section{Conflict of Interest Statement}

The authors declare that the research was conducted in the absence of any commercial or financial relationships that could be construed as a potential conflict of interest.

\section{Funding}


None.

\section{Acknowledgements}

The authors are grateful to Tincontro Associazione Genitori per la Neonatologia Varese for its contributions to the development of this research.

\section{References}

1. Alipour Z, Eskandari N, Ahmari-Tehran H, Eshagh-Hossaini SK, Sangi S. Effects of music on physicological and behavioral responses of premature newborns: A randomized controler trial. Complement Ther Clin Pract (2013) 19: 128-32. doi: 10.1016/j.ctcp.2013.02.007

2. Amini E, Rafiei P, Zarei K, Gohari M, Hamidi M. Effetct of lullaby and classical music on physiologic stability of hospitalized preterm newborns: A randomized trial. J Neonatal Perinatal Med (2013) 6: 295-301. doi: 10.3233/NPM-1371313

3. Loewy J, Stewart K, Dassler AM, Telsey A, Homel P. The effects of music therapy on vital signs, feeding, and sleep in premature newborns. Pediatrics (2013) 131: 902-18. doi: 10.1542/peds.20121367

4. Loewy J. NICU music therapy: song of kin as critical lullaby in research and practice. Ann N Y Acad Sci (2015) 1337: 178-85. doi: 10.1111/nyas. 12648

5. Lubetzky R, Mimouni FB, Dollberg S, Reifen R, Ashbel G, Mandel D. Effect of Music by Mozart on Energy Expenditure in Growing Preterm Newborn. Pediatrics (2010) 125: 24-28. doi: 10.1542/peds.2009-0990

6. Pineda R, Guth R, Herring A, Reynolds L, Oberle S, Smith J. Enhancing sensory experiences for very preterm newborns in the NICU: an integrative review. J Perinatol (2017) 37: 323-332. doi: 10.1038/jp.2016.179

7. Standley J. Music Therapy for the neonate review. Newborn Nurs Rev (2010) 1: 211-216.

8. Van der Heijden MJ, Oliai-Araghi S, Jeekel J, Reiss IK, Hunink MG, Van D. Do Hospitalized Premature Newborns Benefit from Music Interventions? A Systematic Review of Randomized Controlled Trials. PLoS One (2016) 11: e0161848. doi:10.1371/journal.pone.016184.

9. Bieleninik $Ł$, Ghetti C, Gold C. Music Therapy for Preterm Newborns and Their Parents: A Metaanalysis. Pediatrics (2016) 138: e20160971. doi: 10.1542/peds.2016-0971

10. Haslbeck FB. Music from the very beginning- a Neuroscience-Based Framework for music as therapy for preterm infants and their parents. Frontiers Behavioral Neuroscience (2018) 12: 112

11. Standley J. Music therapy research in the NICU: an updated meta-analysis. Neonatal Netw (2012) 31 : 311-6. doi: 10.1891/0730-0832.31.5.311

12. American Academy of Pediatrics, Committee on Environmental Health: Noise: a hazard for the fetus and newborn. Pediatrics (1997) 100: 724-727. 
13. Arnon S, Diamant C, Bauer S, Regev R, Sirota G, Litmanovitz I. Maternal singing during kangaroo care led to autonomic stability in preterm infants and reduced maternal anxiety. Acta Paediatr (2014) 103: 1039-44. doi: 10.1111/apa.12744

14. Task Force of The European Society of Cardiology and The North American Society of Pacing and Electrophysiology. Heart rate variability. Standards of measurement, physiological interpretation, and clinical use. European Heart Journal (1996) 17: 354-381

15. Nöcker-Ribaupierre M. Guidelines for Music Theray Practice in Pediatric Care. Chapter: Premature Infants. Barcelona Publishers (2013) 106: 66-116

16. Schwilling D, Vogeser M, Kirchhoff F, Schwaiblmair F, Boulesteix AL, Schulze A, et al. Live music reduces stress levels in very low-birthweight infants. Acta Paediatr (2015) 104: 360-7. doi:

10.1111/apa.12913

\section{Tables}

Table 1. Demographic characteristics of the patients

\begin{tabular}{|c|c|c|c|c|c|}
\hline & & $\begin{array}{l}\text { All } \\
\mathrm{N}=\mathbf{2 7}\end{array}$ & $\begin{array}{l}\text { ELBW Group } \\
N=13\end{array}$ & $\begin{array}{l}\text { VLBW Group } \\
\mathrm{N}=14\end{array}$ & $P^{*}$ \\
\hline Gender of neonates & (males/females) & $18 / 9$ & $8 / 5$ & $10 / 4$ & 0.586 \\
\hline $\begin{array}{l}\text { Gestational age } \\
\text { (weeks) }\end{array}$ & $\begin{array}{l}\text { Mean } \pm \text { SD } \\
\text { (Range) }\end{array}$ & $\begin{array}{c}29,15 \pm \\
1,77 / \\
29,25 \pm \\
1,89\end{array}$ & $\begin{array}{l}25.77 \pm 1.16 \\
(23-27) *\end{array}$ & $\begin{array}{l}29.71 \pm 1.38 \\
(28-32)\end{array}$ & $<0.001 *$ \\
\hline Birth weight (g) & $\begin{array}{l}\text { Mean } \pm \text { SD } \\
\text { (Range) }\end{array}$ & $\begin{array}{c}1030,53 \pm \\
381,82 / \\
962,5 \pm \\
311,2073\end{array}$ & $\begin{array}{l}792.69 \pm 222.79 \\
(420-1095)\end{array}$ & $\begin{array}{l}1191.93 \pm 196.95 \\
(880-1490)\end{array}$ & $<0.001$ \\
\hline **APGAR 1' & Mean \pm SD & $\begin{array}{c}5,5 \pm \\
2,14 / \\
\\
4,81 \pm \\
2,46\end{array}$ & $3.69 \pm 2.06$ & $6.5 \pm 1.56$ & 0.001 \\
\hline APGAR 5' & Mean \pm SD & $\begin{array}{c}7,78 \pm \\
1,30 / \\
6,61 \pm \\
2,19\end{array}$ & $6.54 \pm 1.81$ & $8.07 \pm 0.57$ & 0.006 \\
\hline
\end{tabular}

*P-value for Fisher's Exact test, Student's T-test or Mann-Whitney test for difference between ELBW and VLBW 
**APGAR A $\rightarrow$ Appearance, $P \rightarrow$ Pulse, G $\rightarrow$ Grimace, A $\rightarrow$ Activity, R $\rightarrow$ Respiratory effort Score Journal of the American Medical Association (JAMA)

Table 2.

Difference between pop-rock, pop-rap and classical music

\begin{tabular}{|c|c|c|}
\hline POP-ROCK & POP-RAP & CLASSICAL \\
\hline ADAGIO-ALLEGRETTO & ADAGIO-ALLEGRETTO & ANDANTINO-ADAGIO \\
\hline $\begin{array}{l}\text { Lively, regular in the verse, change of } \\
\text { tempo and rhythmic-harmonic } \\
\text { variation in the refrain }\end{array}$ & $\begin{array}{l}\text { Sustained regular, repetitive, } \\
\text { same time, no variations in } \\
\text { refrain }\end{array}$ & ${ }^{\circ}{ }^{\circ}$ TEMPO \\
\hline \multirow[t]{2}{*}{$\begin{array}{l}\text { Contrasting Rock Song Structure: } \\
\text { AABACA. Intro, Strofa, Ponte, } \\
\text { Ritornello Rhythmic-harmonic } \\
\text { variation. Melody and against melody }\end{array}$} & \multirow[t]{2}{*}{$\begin{array}{l}\text { Song structure Simple Pop: A B } \\
\text { A - Harmonic range 1-4-5-1- } \\
\text { Intro, Strofa, Bridge, Chorus } \\
\text { equal harmonically }\end{array}$} & $\begin{array}{l}\text { ANDANTINO KV } 299 \|^{\circ} \\
\text { TEMPO, F+, form is A-B- } \\
\text { C-D-C-B-A, Repetitive, } \\
\text { Harp and flute Solo. }\end{array}$ \\
\hline & & $\begin{array}{l}\text { ADAGIO KV } 622 \text { II }^{\circ} \\
\text { TEMPO, D+, form is A-B- } \\
\text { A', Repetitive, clarinet } \\
\text { solo. }\end{array}$ \\
\hline Male voice: Vasco Rossi & Male voice: Lorenzo Cherubini & No voice \\
\hline Voice 2 OCTAVE BARITONO & Voice 1 OCTAVE BARITONO & $\begin{array}{l}\text { SYMPHONY } \\
\text { ORCHESTRA }\end{array}$ \\
\hline $\begin{array}{l}\text { Symphony orchestra, male voice, } \\
\text { piano, harp, drums, chorus, timpani, } \\
\text { percussion }\end{array}$ & $\begin{array}{l}\text { male voice, electric bass, } \\
\text { electric and acoustic guitar, } \\
\text { piano, drums and percussion, } \\
\text { accordion, string orchestra }\end{array}$ & $\begin{array}{l}\text { Symphony orchestra, } \\
\text { harp, flute, clarinet solo. }\end{array}$ \\
\hline $\begin{array}{l}\text { ALBA CHIARA - Key C+- } \\
\text { ALLEGRETTO-5'-Tempo 4/4 }\end{array}$ & $\begin{array}{l}\text { PER TE- Key C+- } \\
\text { ALLEGRETTO- 5'-Tempo 4/4 }\end{array}$ & \multirow{2}{*}{$\begin{array}{l}\text { MOZART KV 299- } \\
\text { ANDANTINO-II TEMPO- } \\
\text { Key F+ - Tempo 3/4 } \\
\text {-7'30" }\end{array}$} \\
\hline $\begin{array}{l}\text { ANIMA FRAGILE- Key D+- ADAGIO-5'- } \\
\text { Tempo } 4 / 4\end{array}$ & $\begin{array}{l}\text { A TE- Key C+ - ADAGIO-5'- } \\
\text { Tempo } 4 / 4\end{array}$ & \\
\hline $\begin{array}{l}\text { UNA CANZONE PER TE - Key- } \\
\text { ADAGIO-5'-Tempo 4/4 }\end{array}$ & $\begin{array}{l}\text { BACIAMI ANCORA- Key Eb+ } \\
\text { ALLEGRETTO -5'-Tempo } 12 / 8\end{array}$ & $\begin{array}{l}\text { MOZART KV 622- } \\
\text { ADAGIO - II TEMPO- Key } \\
\text { D+ - Tempo 3/4 -7'30" } \\
\text { min }\end{array}$ \\
\hline $\begin{array}{l}\text { Teatro La Scala String Orchestra, } \\
\text { Album: L'altra metà del cielo. }\end{array}$ & $\begin{array}{l}\text { Acoustic version; Album: Back } \\
\text { up. }\end{array}$ & $\begin{array}{l}\text { Helms } \mathrm{M}^{\circ} \text { Karayan } \\
\text { (conductor) Galmay } \\
\text { (piano) Berliner } \\
\text { Orchestra }\end{array}$ \\
\hline
\end{tabular}


Table 3.

HRV measurements LF/HF values in 14 VLBW neonates. Music phases and post music vs before music. Wilcoxon test for paired data was applied.

\begin{tabular}{|lllllll|}
\hline Parameter & $\begin{array}{l}\text { Baseline } \\
(0-5 \mathrm{~min})\end{array}$ & $\begin{array}{l}\text { Phase 1 } \\
(5-10)\end{array}$ & $\begin{array}{l}\text { Phase 2 } \\
(10-15)\end{array}$ & $\begin{array}{l}\text { Phase3 } \\
(15-20)\end{array}$ & $\begin{array}{l}\text { Post } \\
(20-25)\end{array}$ & P \\
\hline $\begin{array}{l}\text { LF/HF With Classic M. } \\
\text { Mean } \pm \text { SD }\end{array}$ & $4,79 \pm 2,65$ & $5,76 \pm 2,29$ & $5,83 \pm 4,13$ & $5,38 \pm 3,83$ & $4,55 \pm 2,33$ & n.s. \\
LF/HF With POP-rap Music & $6,03 \pm 2,80$ & $6,36 \pm 6,74$ & $7,56 \pm 4,04$ & $6,84 \pm 4,55$ & $7,56 \pm 5,97$ & n.s. \\
Mean \pm SD & & & & & & \\
\hline $\begin{array}{l}\text { LF/HF With pop-Rock Music } \\
\text { Mean } \pm \text { SD }\end{array}$ & $6,14 \pm 3,16$ & $5,94 \pm 4,41$ & $5,51 \pm 3,56$ & $5,91 \pm 1,96$ & $5,81 \pm 2,17$ & n.s. \\
\hline $\begin{array}{l}\text { LF/HF With No Music } \\
\text { Mean } \pm \text { SD }\end{array}$ & $6,03 \pm 2,53$ & $7,79 \pm 7,25$ & $6,19 \pm 4,02$ & $7,38 \pm 7,97$ & $5,97 \pm 3,36$ & n.s. \\
\hline
\end{tabular}


Table 4.

HRV measurements in 13 ELBW neonates. Music phases and post music vs before music. Wilcoxon test for paired data was applied.

\begin{tabular}{|c|c|c|c|c|}
\hline & & No music & & \\
\hline & & LF & HF & LF/HF \\
\hline Baselin€ & & $89,02 \pm 6,45$ & $10,97 \pm 6,45$ & $11,23 \pm 6,50$ \\
\hline Silence & $0-5 \mathrm{~min}$ & $89,85 \pm 5,03$ & $10,14 \pm 5,03$ & $10,61 \pm 4,29$ \\
\hline & $5-10 \mathrm{~min}$ & $90,00 \pm 4,61$ & $9,38 \pm 4,37$ & $11,43 \pm 5,02$ \\
\hline & $10-15 \mathrm{~min}$ & $88,58 \pm 6,40$ & $10,58 \pm 4,48$ & $10,41 \pm 5,65$ \\
\hline post mu & & $89,68 \pm 3,35$ & $10,01 \pm 2,92$ & $10,11 \pm 4,68$ \\
\hline & & Pop-Rock ${ }^{1}$ & & \\
\hline & & LF & HF & LF/HF \\
\hline Baselin€ & & $83,69 \pm 7,64$ & $16,30 \pm 7,64$ & $6,57 \pm 3,66$ \\
\hline Music & $0-5 \min$ & $83,85 \pm 6,76$ & $16,14 \pm 6,76$ & $6,48 \pm 3,72$ \\
\hline & $5-10 \min$ & $82,18 \pm 13,83$ & $17,81 \pm 13,83$ & $8,14 \pm 7,40$ \\
\hline & $10-15 \min$ & $88,22 \pm 4,76$ & $11,77 \pm 4,76$ & $8,59 \pm 3,32$ \\
\hline Post mu & & $86,15 \pm 6,64$ & $14,79 \pm 5,56$ & $6,87 \pm 3,28$ \\
\hline & & Pop-Rap ${ }^{2}$ & & \\
\hline & & LF & HF & LF/HF \\
\hline Baselin€ & & $90,79 \pm 5,16$ & $9,20 \pm 5,16$ & $14,43 \pm 11,30$ \\
\hline Music & $0-5 \mathrm{~min}$ & $87,26 \pm 9,61$ & $13,48 \pm 9,05$ & $12,69 \pm 14,28$ \\
\hline & $5-10 \mathrm{~min}$ & $84,89 \pm 7,24$ & $15,10 \pm 7,24$ & $7,53 \pm 5,01$ \\
\hline & $10-15 \mathrm{~min}$ & $83,32 \pm 9,02 *$ & $16,67 \pm 9,02^{\star \star}$ & $7,29 \pm 5,42^{\star \star *}$ \\
\hline Post mu & & $87,65 \pm 5,90$ & $12,34 \pm 5,90$ & $8,41 \pm 3,34$ \\
\hline & & Classic Musi & & \\
\hline & & LF & HF & LF/HF \\
\hline Baselin€ & & $82,54 \pm 11,06$ & $17,45 \pm 11,06$ & $7,111 \pm 5,05$ \\
\hline Music & $0-5 \mathrm{~min}$ & $84,02 \pm 8,76$ & $15,97 \pm 8,76$ & $7,95 \pm 6,26$ \\
\hline & $5-10 \mathrm{~min}$ & $84,21 \pm 10,82$ & $15,78 \pm 10,82$ & $8,34 \pm 5,86$ \\
\hline & $10-15 \mathrm{~min}$ & $84,71 \pm 8,69$ & $15,28 \pm 8,69$ & $7,92 \pm 4,94$ \\
\hline
\end{tabular}


1 Pop-rock: Vasco Rossi, Alba Chiara (C+), Anima fragile (D-), Una canzone per te (c+); Teatro La Scala String Orchestra, Album: L'altra metà del cielo.

2 Pop-rap: Jovanotti, A te (A+), Per te (C+), Baciami ancora (Eb+); Acoustic version; Album: Back up.

3 Classic Music: W.A. Mozart, k299 (C+) Adagio, k622 (A+) Adagio, Helms M Karayan (conductor) Galmay (piano) Berliner Orchestra

*statistically significantly different from baseline measurement $(P=0,0010)$

** statistically significantly different from baseline measurement $(P=0,009)$

*** statistically significantly different from baseline measurement $(P=0,007)$

\section{Figures}




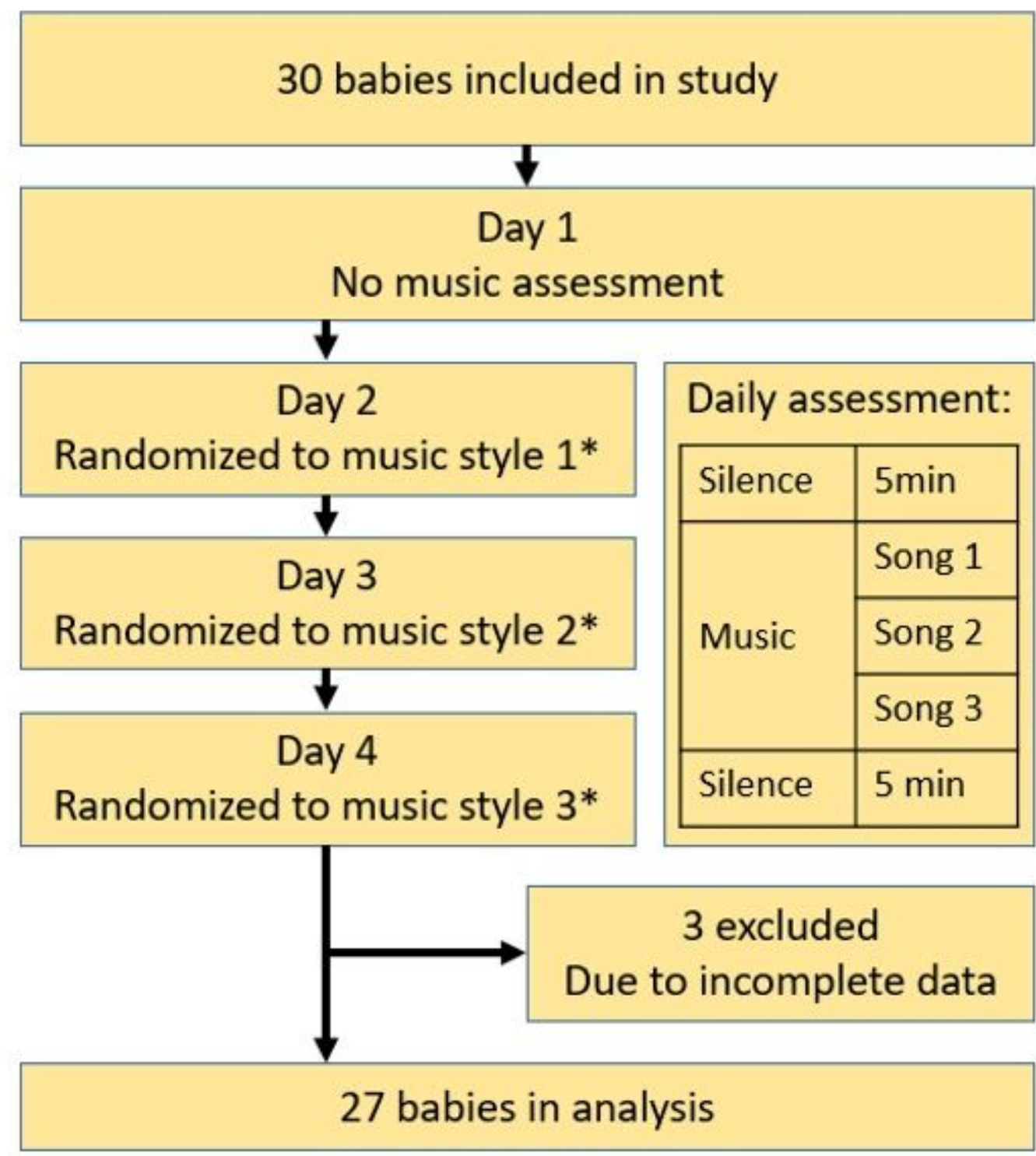

*Order of music style was randomly decided and included: Soft-Pop-Rap, Soft Rock or Classical Music

Figure 1

Enrolment, Randomization, and Retention of Study Participants. 